\title{
Convergence and covering on graphs for wait-free robots
}

\author{
Armando Castañeda ${ }^{1 *}$, Sergio Rajsbaum ${ }^{1}$ and Matthieu Roy ${ }^{2}$
}

\begin{abstract}
The class of robot convergence tasks has been shown to capture fundamental aspects of fault-tolerant computability. A set of asynchronous robots that may fail by crashing, start from unknown places in some given space, and have to move towards positions close to each other. In this article, we study the case where the space is uni-dimensional, modeled as a graph G. In graph convergence, robots have to end up on one or two vertices of the same edge. We consider also a variant of robot convergence on graphs, edge covering, where additionally, it is required that not all robots end up on the same vertex. Remarkably, these two similar problems have very different computability properties, related to orthogonal fundamental issues of distributed computations: agreement and symmetry breaking. We characterize the graphs on which each of these problems is solvable, and give optimal time algorithms for the solvable cases. Although the results can be derived from known general topology theorems, the presentation serves as a self-contained introduction to the algebraic topology approach to distributed computing, and yields concrete algorithms and impossibility results.
\end{abstract}

Keywords: Robot gathering, Agreement, Symmetry breaking, Shared memory, Wait-freedom, Combinatorial topology

\section{Introduction}

The family of robot convergence tasks plays a fundamental role in the theory of fault-tolerant distributed computing [21]. It is used to prove the wait-free computability theorem [25] that characterizes the tasks that are wait-free solvable in a read/write shared memory environment, and is intimately related to the simplicial approximate agreement theorem of topology. Roughly speaking, asynchronous communicating robots cannot coordinate to converge to a single point because consensus is impossible in the presence of even a single crash failure [17], but robots can move towards points which are arbitrarily close to each other, using a solution to approximate agreement [16]. Robots can also converge in Euclidean space; see [28] for a recent treatment of a basic multi-dimensional robot convergence task tolerating Byzantine faults, including a discussion of applications to robots, distributed voting and optimization problems, as well as further related references. Various other

*Correspondence: armando.castaneda@im.unam.mx

'Instituto de Matemáticas, UNAM, Ciudad Universitaria, 04510 Mexico city, Mexico

Full list of author information is available at the end of the article applications and specific robot convergence tasks appear in, e.g., $[8,22,23,27,30]$.

In a robot convergence problem, a collection of $n$ robots are placed in points of a given space, $\mathcal{K}$, which can be of any shape and dimension. The robots know $\mathcal{K}$, but they do not know on which point of $\mathcal{K}$ each robot is initially placed. The goal for the robots is to move to points which are close to each other. The difficulty is that although the robots can communicate reliably with each other, and can jump from one point to any other point of $\mathcal{K}$, the robots are asynchronous and may crash. The combination of asynchrony and failures means that it is impossible to distinguish between a faulty robot that has halted and a robot subject to slow computation [5]. Thus, a robot must continue running its algorithm and decide where to move, independently of which robots it hears from at any given moment (robots do not observe each other positions directly, but only through communication). In particular, in a solo run, where a robot does not hear at all from other robots, the algorithm has to drive the robot to its final position based only on its initial position.

A specific robot convergence task is defined by the space $\mathcal{K}$, and rules $\Delta$ stating restrictions on the regions 
on which the robot should converge. For instance, the space $\mathcal{K}$ could be the $d$-dimensional Euclidean space as in [28], and robots may be required to converge on regions spanned by the convex hull of their initial positions; if all start on the same point, they should remain there, and if all start in two points, they should converge to points close to each other along the straight line connecting the two points. Another example is the loop agreement task [23], where there is a given loop in the space $\mathcal{K}$, and three given distinguished points $v_{1}, v_{2}, v_{3}$ on the loop. The robots are placed on any of these three points. If the robots start on the same point $v_{i}$, they should remain there, if they start on two points, they should converge on the loop segment connecting these two points. If they start on the three different points, they can converge anywhere in $\mathcal{K}$, as long as they end up being close to each other. Whether a specific robot convergence task is solvable depends on the space $\mathcal{K}$ and the convergence rules $\Delta$. Arguably, the most basic (wait-free) unsolvable convergence task is two-set agreement for three processes [14], which is an instance of loop agreement where $\mathcal{K}$ is a cycle of three edges [23]. Stated using this terminology, two-set agreement for three processes corresponds to robots starting in any of the corresponding three vertices, and having to decide on at most two of the initial vertices.

\section{Robot convergence problems on graphs}

We are interested in studying robot convergence problems in the case the space $\mathcal{K}$ is 1 -dimensional. As usual in combinatorial topology, we consider a discretization of the space, and represent it by a graph $G$, where two vertices are defined to be close to each other if and only if they belong to the same edge (the corresponding points can be as close as desired, by considering a subdivision of the space as fine as needed). In the graph convergence problem, robots may start on any of the vertices of the graph and must end up on vertices of the same edge. If they all start in close enough positions, they should stay there: if they start on vertices of an edge, they should decide vertices of this edge. Otherwise, they can decide on vertices of any edge.

We introduce a related problem, edge covering, where the robots have to end in positions close to each other, but not all on top of each other. Thus, while both problems require to reach a form of agreement, edge covering additionally requires symmetry breaking, as robots cannot all decide the same vertex.

Coordination problems in distributed computing can be about reaching agreement, often referred to as colorless problems [8] such as consensus, loop agreement, set agreement, graph convergence, or more generally robot convergence, or they can deal with reaching disagreement, which is usually much more difficult to analyze [19] as in weak symmetry breaking $[11,19,25]$, renaming $[4,12]$ or committee decision [13].

\section{Summary of results}

The first aim of the paper is to study two basic robot convergence problems in a graph, and to expose differences between reaching agreement and symmetry breaking. We formally define and study the graph convergence and edge-covering problems. We give a full characterization of the graphs on which these problems can be solved and provide algorithms where the robots gradually move until they solve the problem. Our results are the following (summarized in Table 1):

1. Graph convergence. For the case of two robots, graph convergence can be solved iff $G$ is connected. If the number of robots is $n \geq 3$, then graph convergence is solvable iff $G$ is a tree.

2. Edge covering. For the case of two robots, edge covering can be solved iff $G$ is connected and contains an odd-length cycle. If the number of robots is $n \geq 3$, then edge covering is unsolvable, whatever the graph $G$.

The second aim of the paper is to provide a selfcontained introduction to the topological approach to distributed computing [21]. The characterization of graph convergence solvability can in principle be derived from existing theorems (e.g., Theorem 4.3.1 of the book [21]) which, roughly speaking, implies that graph convergence has a solution iff there is a continuous map from a given space to $G$ (more details in "The topology of graph convergence" section). Our algorithms explain how such a map is constructed, and our impossibility results explain why there is no such map when $G$ is not connected or is not acyclic. Similarly, our edge covering results can in principle be derived from the Asynchronous Computability Theorem [25] that requires additionally the continuous map to preserve identifiers of participants (because edge covering is not colorless). The topological approach to distributed computing is useful to prove time complexity results, in addition to computability results [26], and we also illustrate this aspect of the theory here. We hope our algorithms and impossibility results provide intuition

Table 1 Summary of results

\begin{tabular}{lll}
\hline & Two robots & $n \geq 3$ robots \\
\hline Graph convergence & $\begin{array}{l}\text { G is connected } \\
\text { Theorem 2 }\end{array}$ & G is a tree \\
Edge covering & Theorem 1 \\
& odd-lenght cycle & Unsolvable \\
& Theorem 3 & Lemma 6 \\
\hline
\end{tabular}


and shed light on these topological theorems, illustrating why topological properties are so intimately related to distributed algorithms.

\section{Related work}

Distributed algorithms for robots is a very active research area (see e.g., [15] for a recent work and further references), and in particular problems about robot convergence, gathering at a single location, and scattering to different locations have been widely studied. Less work has been devoted to fault-tolerant algorithms, and mostly in the plane. Gathering algorithms for the case where at most one robot may crash, or behave in a Byzantine way, was proposed in [1], and for multiple crash failures in [10]. However, we are not aware of the use of algebraic topology techniques in the style of [21] (work about computing topological properties of a space is of a different nature, e.g., [6]). In our setting, gathering is impossible ("Impossibility results" section) because, in contrast to other settings, robots cannot observe directly the positions of other robots, they need to communicate with each other to find them. Notice that our two-robot graph convergence algorithms can be extended for any number of robots tolerating one failure using BG simulation [8].

\section{Outline of the paper}

After introducing the model of computation in "Model of computation" section, we formally define the graph convergence problem and present two round-optimal solutions to it in "The graph convergence problem" section: an algorithm that solves graph convergence on trees, for any number of processes, and an algorithm that solves graph convergence on any connected graph, for two processes. Then, in "The edge-covering problem" section, we define the edge-gathering problem and present a roundoptimal solution for two processes for on any graph. As we shall see in "Impossibility results" section, these are the only graphs where these problems are solvable. A topological perspective of our results is in "A topological perspective" section, where also the optimality of our algorithms is proven.

\section{Model of computation}

We assume a standard distributed computing model (see, e.g., $[5,21]$ for additional details) where $n$ robots, $p_{1}, \ldots, p_{n}$, are sequential processes (state machines), that run asynchronously, namely, the time between any two consecutive steps of the same robot is arbitrarily long. Robots are independent from each other, and any number of them may fail by crashing at any time (and cannot recover). We use the terms interchangeably robots or processes. They communicate by atomically writing and reading single-writer/multi-reader registers.
Robots move on a graph $G$. We assume robots know the graph, and can communicate with each other their current vertex positions using the shared memory. Initially, each robot knows its initial vertex in $G$.

A configuration is a vector containing the local state of each robot. An initial configuration is a configuration in which all robots are in their initial states. A step is performed by a single robot, which executes one of its available local operations. The state machine of a robot $p_{i}$ models a local algorithm $A_{i}$ that determines $p_{i}$ 's next step. A distributed algorithm is a collection $\mathcal{A}$ of local algorithms $\mathcal{A}_{1}, \ldots, \mathcal{A}_{n}$. When a process $p_{i}$ reaches a decided state, it stays in its current vertex forever.

An execution $E$ is an infinite alternating sequence of configurations and steps $E=C_{0} s_{0} C_{1} \ldots$, where $C_{0}$ is an initial configuration and $C_{k+1}$ is the configuration obtained by applying step $s_{k}$ to configuration $C_{k}$. The participating robots in an execution are those robots that take at least one step in that execution. Those robots that take a finite number of steps are faulty (sometimes called crashed), while the others are correct (or non-faulty). That is, the correct robots of an execution are those that take an infinite number of steps. Moreover, a non-participating process is a faulty process. A participating process can be correct or faulty. We are interested in wait-free algorithms: in every execution of the system, every correct robot decide a final vertex, regardless of delays and failures of the other robots.

We describe our algorithms using operations that can be implemented wait-free from registers such as immediate snapshots [9]. Each robot $p_{i}$ invokes the operation with an input value $v_{i}$ and obtains a set of input values $S_{i}$, its view, such that the following three properties are satisfied:

1. Self-inclusion: $\forall i: v_{i} \in S_{i}$.

2. Containment: $\forall i, j: S_{i} \subseteq S_{j} \vee S_{j} \subseteq S_{i}$.

3. Immediacy: $\forall i, j: i \in S_{j} \Rightarrow S_{i} \subseteq S_{j}$.

For completeness, we now describe the recursive immediate snapshot algorithm of [18] using only read and write operations. The presentation follows closely that paper and is repeated here for the convenience of the reader. In the algorithm, each process $p_{i}$ with input $v_{i}$, writes the pair $\left\langle p_{i}, v_{i}\right\rangle$, to a shared memory associated to that recursive call $r_{i}$, and reads (one-by-one, in an arbitrary order) the registers of all other processes. A shorthand for the sequence of operations consisting of first writing and then collecting the inputs of all processes is WriteCollect. If the set $s$ of values collected is of size $r_{i}$, it returns this set as a view and terminates the algorithm; else, the process calls the algorithm recursively with parameters $\left(v_{i}, r_{i}-1\right)$. The first call of process $p_{i}$ is with parameters $\left(v_{i}, n\right)$. We stress that in each recursive call, the processes communicate with each other via a new 


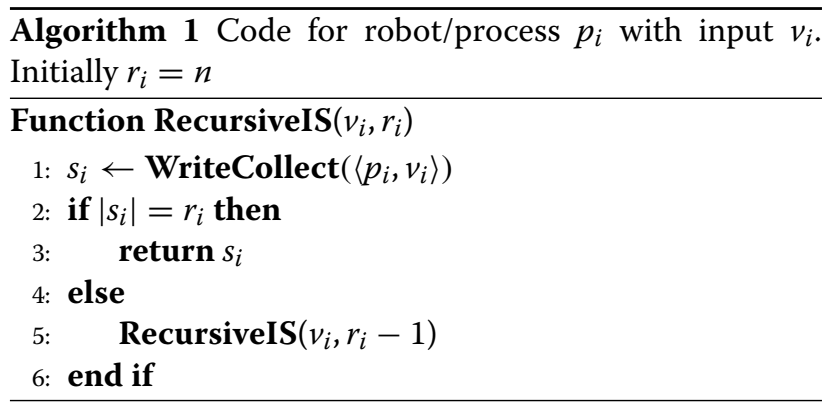

array of single-writer/multi-reader registers, that is used only in that recursive call.

Consider the case of three processes, with inputs 1,2 , and 3, respectively. Interestingly, all their possible outputs (running this algorithm) can be graphically represented through a subdivision of a triangle, as shown in Fig. 1. Each triangle represents a possible execution, and the vertices are labeled with the views (outputs) of each one of the processes at the end of the execution. The fully concurrent execution, where all three processes collect the views of each other, and all terminate the algorithm without a recursive call, is represented by the triangle at the center. This triangle shares an edge with three other triangles. These represent executions where two processes see the inputs of all three processes and terminate without a recursive call, while the first process executes two recursive calls and ends up seeing only its own input. For more processes, all outputs can be represented a subdivision of a higher dimensional object, e.g., for four proceses, a subdivision of tetrahedron is needed.

\section{The graph convergence problem}

In the graph convergence problem on a graph $G$, each robot starts with an input vertex of $G$ and, after communicating with other robots, has to eventually decide a vertex such that the following two properties are satisfied:

- Agreement: The collection of decided vertices belong to a single edge of $G$.

- Validity: If the input vertices are equal, then each process must decide this vertex; if the input vertices span an edge, then each process must decide a vertex of that edge.

Notice that this is exactly the definition of a robot convergence task [21] specialized to the case of graphs. In the graph gathering problem, the agreement property is replaced by requiring that the decided vertices are equal. Thus, in principle one could use general theorems in [21] to identify cases where the problem is solvable; we elaborate further on this point in "A topological perspective" section. The optimality of the two algorithms in the next two sections follows from arguments similar to those in [26]. This is explained in detail in "Round-complexity optimality" section.

\section{Graph convergence on trees}

Recall that the eccentricity of a vertex $v$ is defined as the greatest distance from $v$ to any other vertex. A center of a graph is a vertex with minimal eccentricity. The radius

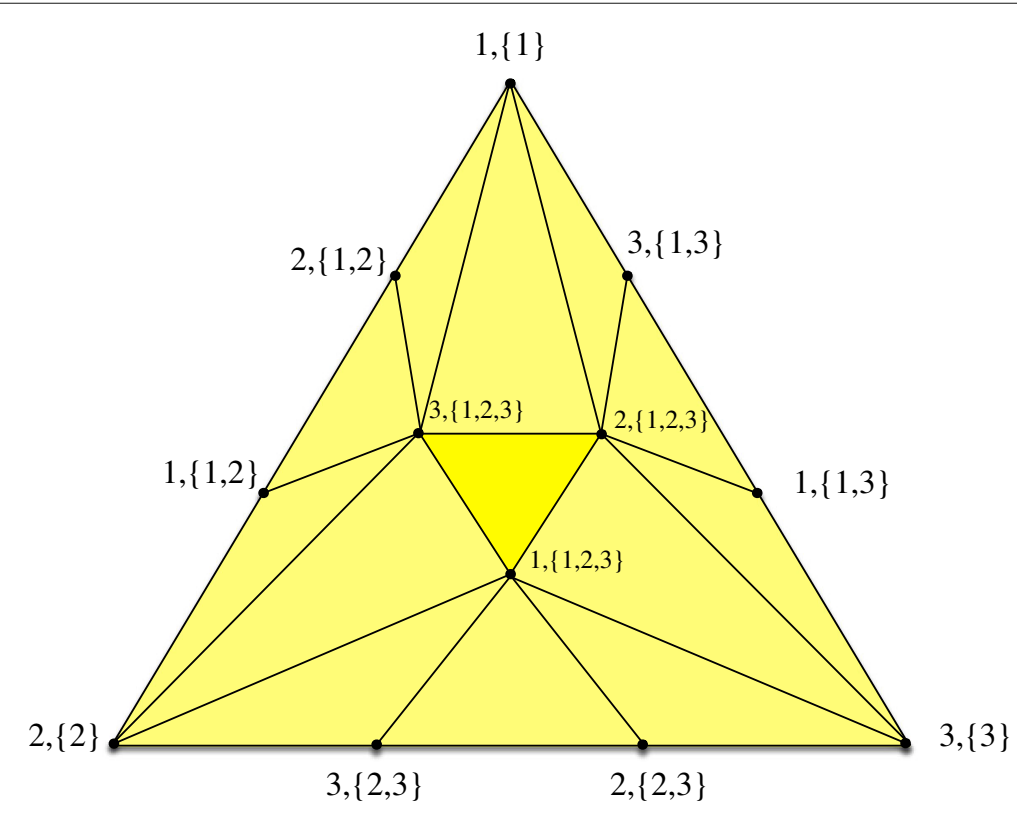

Fig. 1 Graphic description of immediate snapshots. All immediate snapshot views by three processes starting on 1, 2, and 3, respectively 


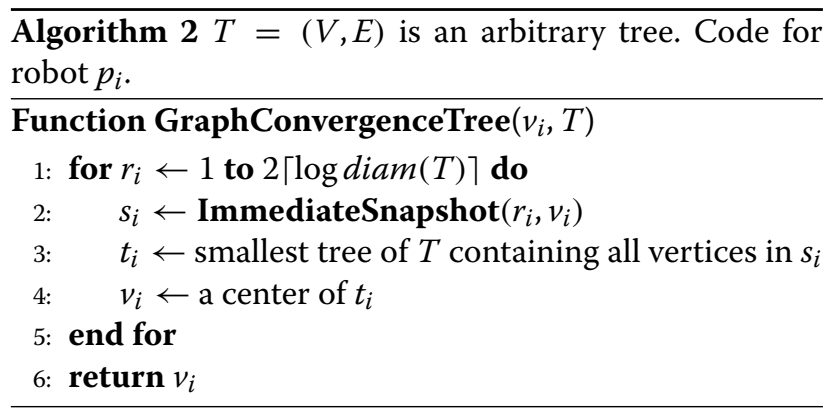

of $G$ is the minimum eccentricity among the vertices of $G$ and the diameter of $G$ is the maximum eccentricity among the vertices of $G$. Denoting the centers of a graph $G$ by center $(G)$, a tree $T$ has $|\operatorname{center}(T)|=1$ or $|\operatorname{center}(T)|=2$. If $\mid$ center $(T) \mid=1$, the tree is called central. If $\mid$ center $(T) \mid=2$, the tree is called bicentral. For any graph $G$, the diameter is at least the radius and at most twice the radius. Trees have the following property, which we will exploit in our graph convergence solution.

Remark 1 For a tree $T$, $\operatorname{diam}(T)=2 \times \operatorname{rad}(T)-1$, if $T$ is bicentral, and $\operatorname{diam}(T)=2 \times \operatorname{rad}(T)$, if $T$ is central.

The algorithm GraphConvergenceTree (Algorithm 2) solves graph convergence on trees for any number of robots. The idea is very simple: robots proceed in a sequence of rounds, and in every round, each robot $p_{i}$ communicate to the others its current vertex $v_{i}$ (its input vertex in the case of the first round) and using the vertices in its snapshot $s_{i}$ (which does not necessarily contain all vertices of the corresponding round, due to asynchrony), $p_{i}$ computes a subtree $t_{i}$ of $T$ and "moves" to a center of $t_{i}$. Thus, processes converge by gradually moving to the center of the trees they see during the computation. In the algorithm, each round has an associated immediate snapshot operation which is indexed with the round number passed to the operation as an input parameter.

Figure 2 depicts the tress that three robots, $p_{1}, p_{2}$, and $p_{3}$, can obtain in a single round of an execution of algorithm GraphConvergenceTree (the execution involves more than those processes). In the example, $p_{1}$ executes the round before $p_{2}$ and $p_{2}$ executes the round before $p_{3}$. The properties of the ImmediateSnapshot primitive imply that the snapshot of $p_{1}$ contains less information than the snapshot of $p_{2}$ (particularly, the snapshot of $p_{1}$ does not contain the position of $p_{2}$ at the beginning of the round) and, similarly, the snapshot of $p_{2}$ contains less information than the snapshot of $p_{3}$. Namely, $s_{1} \subset s_{2} \subset s_{3}$. Each snapshot $s_{i}$ induces a tree $T_{i}$, and thus $T_{1} \subset T_{2} \subset T_{3}$. Finally, the position of each $p_{i}$ for the next round is the center $c_{i}$ of $T_{i}$. Note that the centers induce a spanning tree that is strictly contained in $T_{3}$, the largest tree of the round.

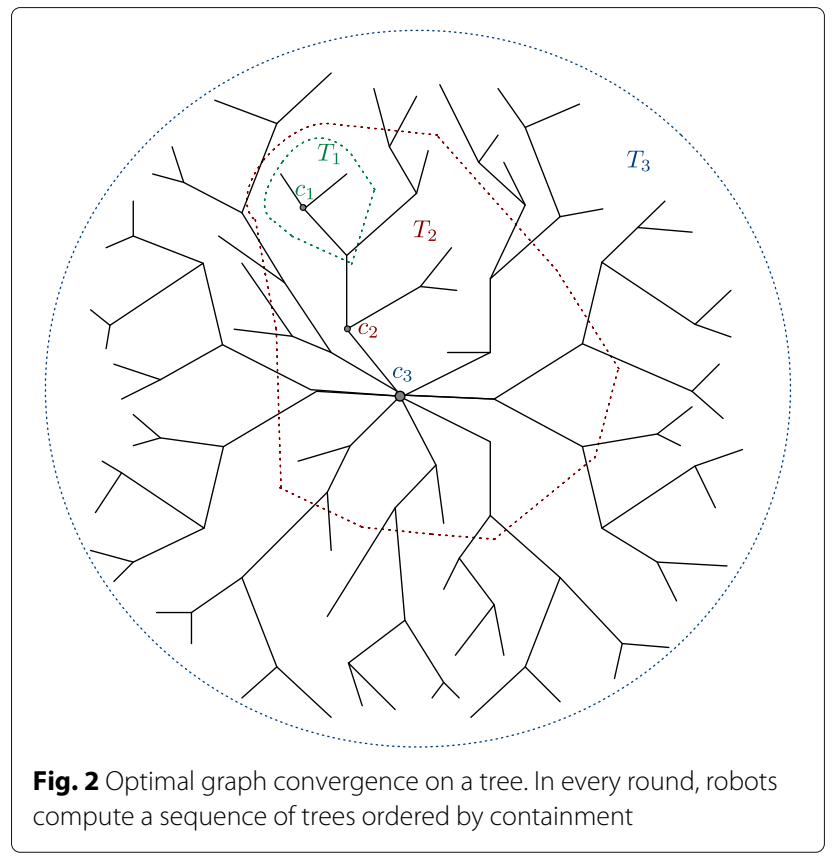

Clearly, every non-crashed robot terminates in GraphConvergenceTree as the number of rounds depends only on the shared tree $T$. It is also easy to see that the algorithm satisfies the validity property of the graph convergence problem: If all the initial vertices already span a vertex, then each non-crashed robot $p_{i}$ returns its initial vertex as its snapshot in every round $t_{i}$, its contains the single initial vertex. Similarly, if all initial vertices span an edge $e$, then $p_{i}$ returns a vertex of $e$ because its snapshot in every round $t_{i}$ is either $e$ or a vertex of $e$.

The correctness of algorithm GraphConvergenceTree follows from the following lemma showing that every round roughly halves the distance between any two vertices, hence implying that the algorithm satisfies the agreement property. For simplicity, in what follows, we only consider executions in which all robots decide a final vertex. Observe that there is no loss of generality by assuming this, as any execution in which every noncrashed robot returns, a vertex can be extended to an execution in which every robot returns a vertex. Intuitively, in the extended execution, we think of "faulty" robots as very slow that take steps only after all "correct" robots are decided.

Lemma 1 For $j=1, \ldots, 2\lceil\log \operatorname{diam}(T)\rceil$, let $v_{i}^{j}$ be the value of $v_{i}$ at the beginning of the $j$-th iteration, and let $T_{j}$ be the smallest subtree of $T$ containing $v_{1}^{j}, \ldots, v_{n}^{j}$. Then, $\operatorname{diam}\left(T_{j+1}\right) \leq\left(\operatorname{diam}\left(T_{j}\right)+1\right) / 2$.

Proof The inclusion property of the ImmediateSnapshot operation implies that the snapshots of the robots in the $j$-th round are ordered by containment, and hence there 
are sets $S_{1} \subset S_{2} \subset \ldots \subset S_{k}=\left\{v_{1}^{j}, \ldots, v_{n}^{j}\right\}$, for some $k \geq 1$, such that, for each robot $p_{i}$, its snapshot in the $j$-th round is equal to some $S_{l}$. Moreover, by the immediacy property, every robot whose vertex is in $S_{l}$ has its snapshot equal to $S_{l}$. For each $S_{l}$, let $R_{l}$ be the smallest subtree of $T_{j}$ containing every vertex in $S_{l}$. Thus, we have that $R_{1} \subset R_{2} \subset \ldots \subset$ $R_{k}=T_{j}$, and consequently, $v_{1}^{j+1}, \ldots, v_{n}^{j+1}$ are nothing else than the centers of these trees, center $\left(R_{1}\right), \ldots$, center $\left(R_{k}\right)$ (see Fig. 2). Hence, $T_{j+1}$ is the smallest subtree of $T_{j}$ containing center $\left(R_{1}\right), \ldots$, center $\left(R_{k}\right)$. Let $R_{m}^{\prime}$ be the smallest subtree of $T_{j}$ containing the first $m$ centers, center $\left(R_{1}\right), \ldots$, center $\left(R_{m}\right)$ (thus $\left.R_{k}^{\prime}=T_{j+1}\right)$. By induction of $l$, one can show that $\operatorname{diam}\left(R_{l}^{\prime}\right) \leq \operatorname{rad}\left(R_{l}\right)$. The base case $l=1$ is obvious as $R_{1}^{\prime}$ is a single vertex. Once we have assumed the claim holds for $l$, to show it holds for $l+1$, it is enough to observe that the distance between any pair center $\left(R_{S}\right)$ and center $\left(R_{t}\right)$, distinct both from center $\left(R_{k}\right)$, is at most $\operatorname{rad}\left(R_{l}\right)$, by induction hypothesis, which is at most $\operatorname{rad}\left(R_{j+1}\right)$, by definition; and the distance from center $\left(R_{l+1}\right)$ to any other center $\left(R_{s}\right)$ is at most $\operatorname{rad}\left(R_{l+1}\right)$ by definition. Thus, when $l=k$, we have that $\operatorname{diam}\left(T_{j+1}\right) \leq \operatorname{rad}\left(T_{j}\right)$. By Remark 1 , it follows that $\operatorname{rad}\left(T_{j}\right) \leq\left(\operatorname{diam}\left(T_{j}\right)+1\right) / 2$, and hence $\operatorname{diam}\left(T_{j+1}\right) \leq$ $\left(\operatorname{diam}\left(T_{j}\right)+1\right) / 2$.

Lemma 1 directly implies that after $O(\log (\operatorname{diam}(T)))$ rounds, all robots end up spanning a vertex or an edge of $T$.

Theorem 1 For any tree T, algorithm GraphConvergenceTree solves graph convergence on $T$ for $n \geq 2$ robots.

\section{Graph convergence for two robots on connected graphs}

Let us now focus on two robots solving graph convergence on a connected graph. In the following, we describe a modification of GraphConvergenceTree, tailored for this case.

In GraphConvergenceTwoRobots (Algorithm 3), we assume the robots know (or deterministically compute) a pre-defined shortest path in $G$ between any pair of vertices; thus if the two vertices are the same, the path is the vertex itself, and if the vertices are adjacent, the path is the edge between them. The two robots first take an immediate snapshot to communicate their input vertices. If a robot $p_{i}$ sees the input of the other, it sets $P_{i}$ to the corresponding precomputed path. Due to the view containment property of immediate snapshots, it cannot be that both $P_{i}$ and $P_{j}$ are equal to $\perp$, and if both are distinct to $\perp$, then $P_{i}=P_{j}$. Then, the robots proceed similarly as in GraphConvergenceTree: they move to the center of the subpath between their current vertices. Observe that

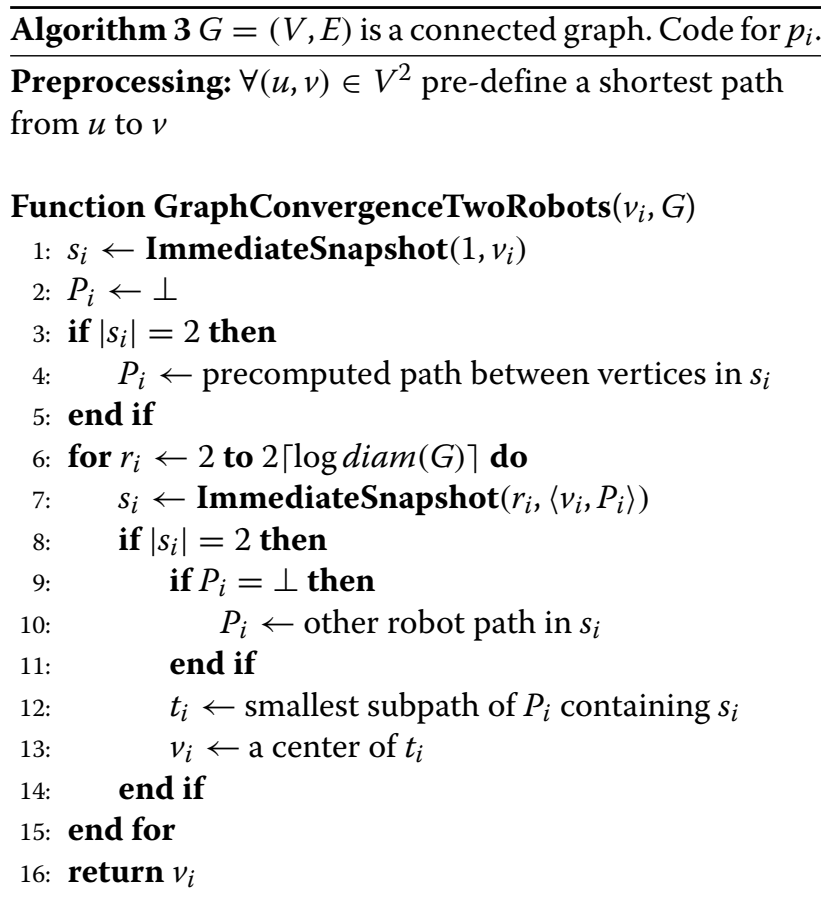

the first round guarantees that the robots move along the precomputed between the initial vertices in all subsequent rounds. Moreover, the path process $p_{i}$ sets in $P_{i}$ in the first round is written in every round because in the case the other process $p_{j}$ goes faster than $p_{i}$ in the first round, it will be impossible to $p_{j}$ to know the initial vertex of $p_{i}$, and hence set $P_{j}$ to the path $P_{i}$ (since a process does not read a shared memory of a previous round).

Theorem 2 For any connected graph G, algorithm GraphConvergenceTwoRobots solves graph convergence on $G$ for two robots.

\section{The edge-covering problem}

This section introduces the edge-covering problem and then presents an algorithm that solves it for two processes on any connected graph $G$ that has a cycle of odd length. Our edge-covering solution, EdgeCoveringTwoRobots (Algorithm 4), is an adaptation of the GraphConvergenceTwoRobots algorithm in the previous section. Surprisingly, the algorithm cannot be generalized for more than two robots; as we shall see, edge covering for three or more processes is impossible on any graph.

In the edge covering problem on a given graph $G$, each robot starts with an input vertex of $G$ and has to eventually decide a vertex such that:

- Agreement: The collection of decided vertices belong to a single edge, and not all decided vertices are equal. 
- Validity: If the initial input vertices span an edge, then each robot must decide a vertex of that edge. If a robot runs alone, it should decide its input vertex.

\section{Edge-covering for two robots on connected graphs with odd length cycles}

Algorithm EdgeCoveringTwoRobots needs a deterministic preprocessing phase that, for any pair of vertices $v_{i}$ and $v_{j}$, computes a simple odd length path from $v_{i}$ to $v_{j}$; if $v_{i}=v_{j}$ then it is needed a simple odd length cycle (without such a cycle, finding such a path is impossible). First, if $\left(v_{i}, v_{j}\right)$ is an edge, then the path from $v_{i}$ to $v_{j}$ is this edge. Otherwise, consider a simple cycle $C=w_{1}, w_{2}, \ldots, w_{x}, w_{1}$ of $G$ of odd length (whose existence is guaranteed by out initial hypotheses). Since $G$ is connected, there are paths $P_{i}$ and $P_{j}$ from $v_{i}$ to $w_{1}$ and from $w_{1}$ to $v_{j}$, respectively. If the length of the composed path $P_{i}-P_{j}$ is odd, we are done, otherwise, the length of the path $P_{i}-C-P_{j}$ must be odd. In any case, for every $v_{i}$ and $v_{j}$, there is a odd length simple path between them. The cycle $C$ and paths $P_{1}$ and $P_{2}$ can be efficiently computed with a classical Breadth First Search. Note that every precomputed path is bicentral.

Now, as in GraphConvergenceTwoRobots, the two robots first take a snapshot to communicate its input vertex to the other and if a robot $p_{i}$ sees the input of the other, it sets $P_{i}$ to the corresponding precomputed path (at least one of $P_{1}$ and $P_{2}$ is distinct from $\perp$ ). Then, the

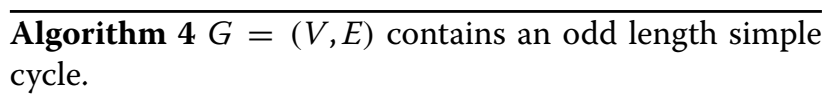

Preprocessing: $\forall(u, v) \in V^{2}$, compute a simple and shortest odd length path of $G$ from $u$ to $v$

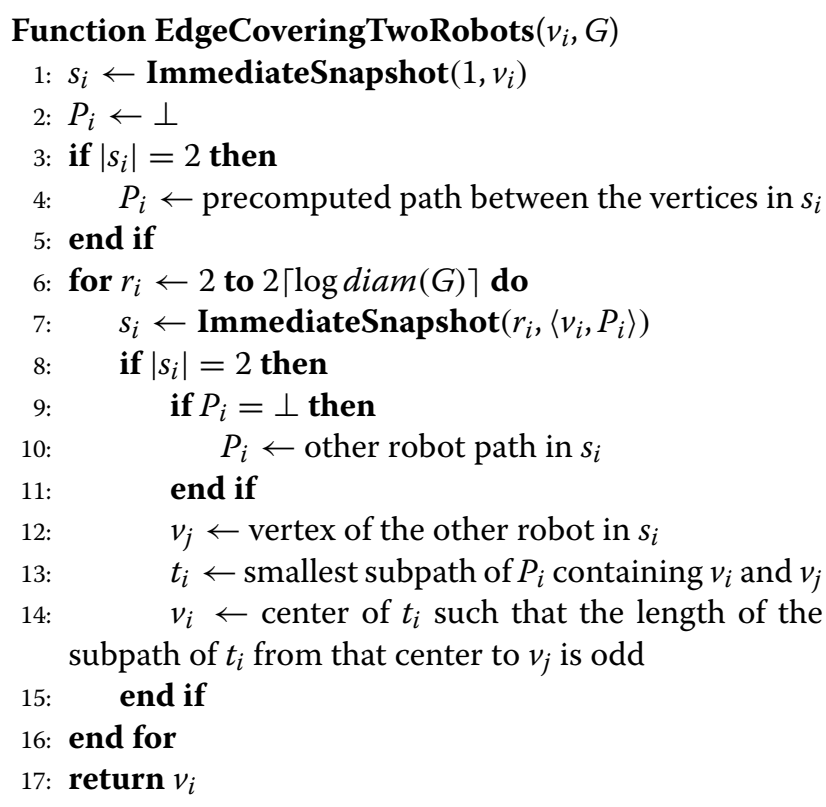

robots move to a center of the subpath between its current vertices. The difference with algorithms in the previous section is that each robot now picks a center that guarantees that the new positions at the beginning of the next round are at odd distance from each other. Figure 3 describes the associated process: if the distance between $v_{1}$ and $v_{2}$ is $2 d+1$ then, depending on the parity of $d$, a robot will move to a position such that the distance to the other is lowered and still odd, independently of whether the other moves or not (in Fig. 3, the top case corresponds to an odd value of $d$, while the bottom case is when $d$ is even). This invariant, which holds at the beginning of any execution because of the precomputed paths (and ultimately because of the existence of an odd length cycle in $G$ ), implies that robots end up at distance exactly one.

Lemma 2 Let $P$ be the precomputed path between the initial vertices $v_{1}$ and $v_{2}$. For $j=1, \ldots, 2\lceil\operatorname{diam}(G)\rceil$, let $v_{1}^{j}$ and $v_{2}^{j}$ be the values of $v_{1}$ and $v_{2}$ at the beginning of the $j$-th iteration, and let $P_{j}$ be the smallest subpath of $P$ containing them. Then, $\left|P_{j+1}\right|$ is odd and less or equal to $\left(\left|P_{j}\right|+1\right) / 2$.

Proof First, observe that $P_{1}=P$, hence, by construction, $\left|P_{1}\right|$ is odd. Thus, we assume $\left|P_{j}\right|$ is odd. Note that $P_{j}$ is a bicentral tree as its length is odd. Let $(u, v)$ be the edge containing its two centers. Let $s_{1}$ and $s_{2}$ be the immediate snapshots of $p_{1}$ and $p_{2}$ in the $j$-th iteration. We have three cases:

- $\left|s_{1}\right|=\left|s_{2}\right|=2$. In this case, both $s_{1}$ and $s_{2}$ contain $v_{1}^{j}$ and $v_{2}^{j}$. Then, both robots $p_{1}$ and $p_{2}$ have $t_{1}$ and $t_{2}$ equal to $T_{j}$. Observe that if the length of subpath of $T_{j}$ from $u$ to $v_{1}^{j}$ is odd (resp. even), then the length of the subpath from $u$ to $v_{2}^{j}$ is even (resp. odd). It similarly happens with $v$ (see Fig. 3). Then, it must be that either $v_{1}^{j+1}=u$ and $v_{2}^{j+1}=v$, or $v_{1}^{j+1}=v$ and $v_{2}^{j+1}=u$. In either case $T_{j+1}=(u, v)$.

- $\left|s_{1}\right|=1$ and $\left|s_{2}\right|=2$. In this case, $s_{1}$ only contains $v_{1}^{j}$ while $s_{2}$ contains $v_{1}^{j}$ and $v_{2}^{j}$. Then, $v_{1}^{j+1}=v_{1}^{j}$ and $v_{2}^{j+1}$ is the center of $P_{j}$ such that the length of the subpath $Q$ from that center to $v_{1}^{j}$ is odd (as explained in the previous case, only one center has that property).

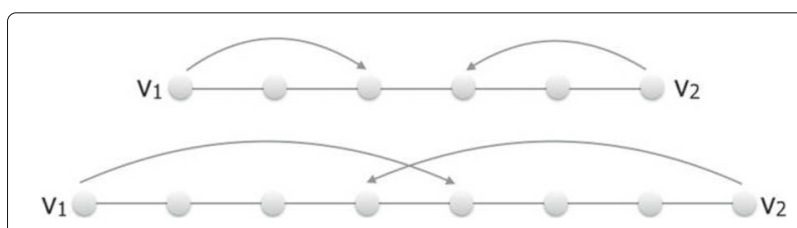

Fig. 3 Edge covering for two robots. Each robot moves to a central vertex that preserves the odd-path path invariant 
Thus, $T_{j+1}=Q$. Remark 1 implies that $Q=\left(\left|T_{j}\right|+1\right) / 2$.

- $\left|s_{1}\right|=2$ and $\left|s_{2}\right|=1$. This case is symmetric to the previous case.

Lemma 2 shows that at the end of EdgeCoveringTwoRobots, the two robots end up on vertices that span an edge of $G$, hence we have the following.

Theorem 3 For any connected graph $G$ containing a simple cycle of odd length, algorithm EdgeCoveringTwoRobots solves the edge covering problem for two robots.

\section{Impossibility results}

In this section, we present a series of impossibility results that fully characterize the solvability of graph convergence and edge covering.

We first show that if $G$ is disconnected, then graph convergence and edge covering are impossible. The reason is that a solution to any of these problems on $G$ can be used to solve wait-free binary consensus, which is known to be impossible $[5,20]$. The following style of proof is known since the first (1-resilient) task characterization results [7].

Lemma 3 If $G$ is disconnected, then graph convergence and edge covering on $G$ are impossible for any number of robots $n \geq 2$.

Proof Assume, for the sake of contradiction, that there is an algorithm $A$ that solves graph convergence on $G$ for $n \geq 2$ robots (the proof is the same when $A$ solves edge covering). Using $A$, we solve binary consensus among $n$ robots, which is known to be impossible [5, 20]. For sake of simplicity, we focus on the binary consensus problem, where each robot proposes either 0 or 1 , and robots are required to decide proposed values so that all decisions are equal.

Let $v_{0}$ and $v_{1}$ be vertices of $G$ belonging to distinct connected components. Let $C_{0}$ be the connected component $v_{0}$ belongs to. We solve binary consensus as follows. Each robot $p_{i}$ with proposal $j \in\{0,1\}$, invokes $A$ with input $v_{j}$. Let $w_{i}$ be the value $A$ outputs to $p_{i}$. Then, $p_{i}$ decides 0 if $w_{i}$ belongs to $C_{0}$ and 1 otherwise.

If all proposal are equal to $j \in\{0,1\}$, then every robot receives $v_{j}$ from $A$, since $A$ is a graph convergence solution. Then, every robot decides $j$, which solves consensus. If robots propose distinct values, then they invoke $A$ with distinct inputs, $v_{0}$ and $v_{1}$. Since $A$ solves graph convergence, it outputs vertices that span a vertex or an edge of $G$. Note that it cannot be that some of these vertices belong to $C_{0}$ and the rest to $G \backslash C_{0}$. Therefore, all robots decides either 0 or 1 and all decisions are the same, which solves consensus.

The following lemma shows that cycles are an obstacle for solving graph convergence and edge covering when the number of robots is greater or equal than three. The structure of the proof is similar to proof of the previous lemma: if there is a solution to a graph with cycles, then one can solve the well-known set agreement problem [14], which has been proved to be unsolvable (see [21]).

Lemma 4 If $G$ has a cycle, then graph convergence and edge covering on $G$ are unsolvable for $n \geq 3$ robots.

Proof By contradiction, suppose that there is an algorithm $A$ that solves graph convergence on $G$ for $n \geq 3$ robots (the proof for edge covering is the same). We use $A$ to solve two-set agreement for three processes hence reaching a contradiction. For the sake of simplicity, we focus on the inputless version of the $(n-1)$-set agreement problem each process $p_{i}$ has as input its index $i$, and every correct process is required to decide an index of a process that participates in the execution such that at most $n-1$ distinct indexes are decided by the processes. It is wellknown that $(n-1)$-set agreement is unsolvable. Thus, $A$ cannot exist because it implies a solution to $(n-1)$-set agreement.

Below we use the following remark that directly follows from the specification of graph convergence and edge covering, which are adaptive by nature.

Remark 2 Let $G$ be a graph and suppose there is an algorithm A that solves graph convergence (edge covering) on $G$ for $n \geq 3$ robots. Then, A solves graph convergence (edge covering) on $G$ for $n-1$ robots.

Therefore, this last remark implies that we can assume $A$ solves graph convergence on $G$ for three robots.

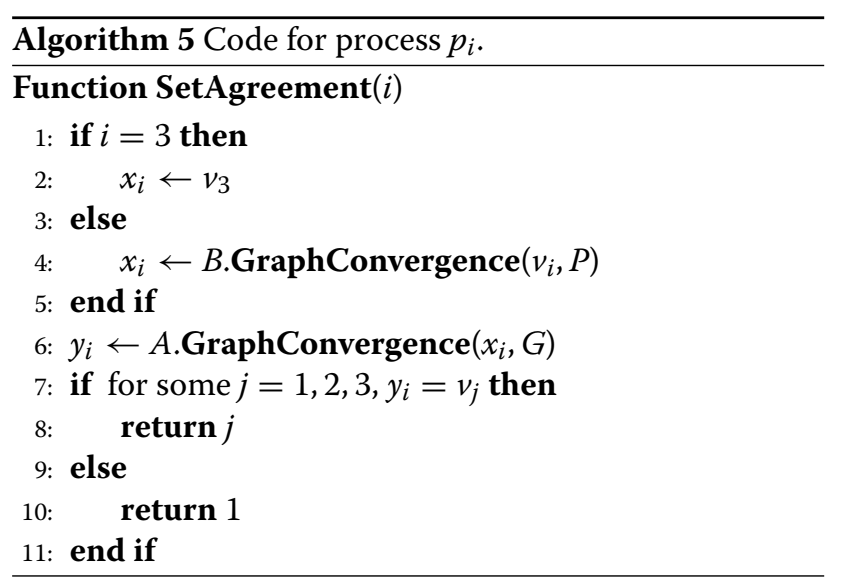


Let $C$ be a simple cycle of $G$ and let $v_{1}, v_{3}, v_{2}$ be three distinct and consecutive vertices of $C$. Let $P$ be the simple odd path obtained by removing $v_{3}$ from $C$ (see Fig. 4). By Theorem 2, let $B$ be an algorithm that solves graph convergence on $P$ for processes $p_{1}$ and $p_{2}$. We use $A$ and $B$ to solve two-set agreement for three robots.

Algorithm 5 solves two-set agreement for three robots, $p_{1}, p_{2}$, and $p_{3}$, using algorithms $A$ and $B$. The idea of the solution is that robots use $A$ to "agree" on a vertex or an edge of $G$, namely, on at most two distinct vertices, and then use these information to return at most two distinct indexes of participating processes. Thus, vertices of $G$ are mapped to indexes of processes: $v_{3}$ is mapped to $3, v_{2}$ is mapped to 2 and the remaining vertices are mapped to 1, as illustrated in Fig. 4. The properties of $A$ make easy to achieve agreement: at most to distinct indexes are decided since $A$ solves graph convergence. What is more complicated to achieve is validity: only indexes from participating processes can be decided. That is the aim of algorithm $B$ and actually the most complicated case is when $p_{1}$ and $p_{2}$ participate: they use $B$ to cover a vertex or an edge of $P$ and these vertices are the inputs the use for $A$; this step guarantees that none of them gets $v_{3}$ from $A$, so each of them returns either 1 or 2 .

We now show that Algorithm 5 is correct.

- Termination. By assumption, $A$ and $B$ terminate in all invocations, thus in every execution, a nonfaulty robot returns a value.

- Validity. We identify three cases, according to the number of robots that participate and decide in a given execution.

- One robot $p_{i}$ participates in an execution. If $p_{i}=p_{3}$, then it invokes $A$ with input $v_{3}$, and consequently obtains $v_{3}$ from it, by validity property of edge convergence. Thus, $p_{3}$ returns 3 .

If $p_{i}$ is either $p_{1}$ or $p_{2}$, it invokes $B$ with input $v_{i}$, and consequently obtains $v_{i}$ from $B$, by

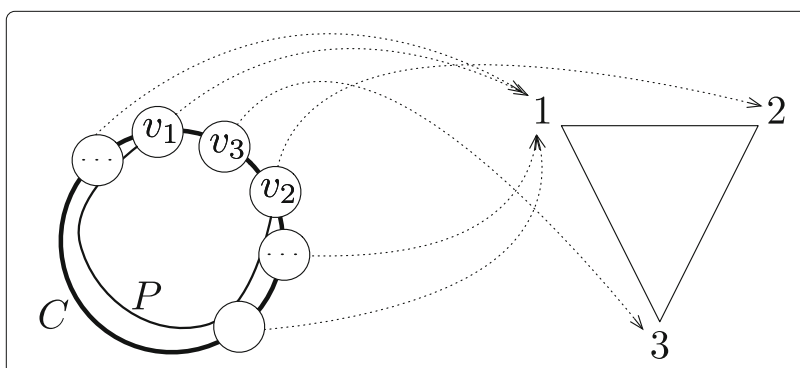

Fig. 4 Impossibility of graph convergence on cycles. Mapping vertices of the cycle $C$ to values for set agreement validity of graph convergence; and hence $p_{i}$ invokes $A$ with $v_{i}$ and obtains $v_{i}$ as well, for the same reason, therefore it returns $i$.

- Two processes $p_{i}$ and $p_{j}$ participate. If $p_{i}=p_{3}$ and $p_{j}$ is either $p_{1}$ or $p_{2}$, then $p_{j}$ invokes solo $B$ with input $v_{j}$, hence it obtains $v_{j}$. Thus, $p_{i}$ and $p_{j}$ invoke $A$ with inputs $v_{i}$ and $v_{j}$, respectively, and thus they obtains these vertices from $A$ since, by assumption, $A$ solves graph convergence and, by definition, these vertices are an edge of $G$. We conclude that $p_{i}$ returns $i$ and $p_{j}$ return $j$.

If $p_{i}=p_{1}$ and $p_{j}=p_{2}$, then they obtain from $B$ two vertices $x_{i}$ and $x_{j}$, respectively, that cover a vertex or an edge of $P$, since $B$ solves graph convergence on $P$. Then, $p_{i}$ and $p_{j}$ get the very same vertices from $A$ since it solves graph convergence on $G$, by assumption. Thus, each of $p_{i}$ and $p_{j}$ returns either 1 or 2 because the only way a process returns 3 is if it gets $v_{3}$ from $G$, but $v_{3} \notin V(P)$.

- The three robots participate. From the pseudocode, it is easy to see that a robot can only decide 1,2 , or 3 . If all processes participate, any of these decisions satisfy validity.

- Agreement. The only interesting case is when the three processes return a value. Since $A$ solves graph convergence on $G$, the values it returns $y_{1}, y_{2}$ and $y_{3}$ to $p_{1}, p_{2}$, and $p_{3}$, respectively, cover a vertex or an edge of $G$, hence at most two distinct indexes are decided.

We conclude that Algorithm 5 solves two-set agreement for three processes, which, as already explained, is a contradiction, from which follows that such an algorithm $A$ cannot exist.

Lemmas 3 and 4 together with Theorems 1 and 2 in "The graph convergence problem" section completely characterize the solvability of graph convergence.

Theorem 4 (Solvability of Graph Convergence) For two robots, graph convergence on a graph $G$ is solvable if and only if $G$ is connected. For three or more robots, graph convergence on $G$ is solvable if and only if $G$ is acyclic.

We now fully characterize the solvability of the edgecovering problem. As we will see, the extra requirement of edge covering that processes always have to cover an edge, precludes solutions for three or more robots, for any graph. The next lemma shows a necessary condition for the solvability of edge covering for two robots. 
Lemma 5 Let $G$ be a graph. If there is an algorithm that solves edge covering on $G$ for two processes, then $G$ is connected and has a simple cycle of odd length.

Proof Let $G$ be any graph and suppose there is an algorithm $A$ that solves edge covering on $G$ for two processes. Lemma 3 implies that $G$ is connected. To show that $G$ has a simple cycle of odd length, suppose the contrary, namely, suppose that $G$ has no simplex cycle of odd length. We will use $A$ to solve weak symmetry breaking (WSB) for two robots [19]. The WSB for two robots is an inputless problem in which each robot has to decide 0 or 1 such that in solo executions, the decision is the same and if the two robots participate, they decide distinct values. It is known that WSB for two robots is unsolvable (see [21]).

Before solving WSB, we observe that $G$ is bipartite since it has no odd length cycles, hence it has a proper vertex binary coloring $c$. To solve WSB, each robot $p_{i}$ invokes $A$ with a fixed vertex $v$ (the same for both robots), and decides $c(w)$, where $w$ is the vertex $A$ outputs to $p_{i}$. Clearly, robots decide 0 or 1 , since $c$ is a binary coloring. In a solo execution of any robot $p_{i}, A$ outputs $v$ to $p_{i}$, by validity of edge covering, and hence it decides $c(v)$. Finally, if the two robots participate, they decide distinct values because $c$ is a binary coloring and $A$ outputs to the robots vertices of $G$ that span an edge. Thus, using $A$, we can solve WSB, which is a contradiction.

Using the previous lemma, we can show that edge covering is unsolvable for three or more processes. The proof is that if there is an algorithm that solves edge covering on a graph $G$ for three or more processes, then, by the adaptive nature of edge covering, this algorithm solves edge covering on $G$ for two processes, and hence $G$ has a cycle, by Lemma 5 . But this contradicts Lemma 4.

Lemma 6 For any graph $G$, there is no algorithm that solves edge covering on $G$ for three or more robots.

Proof Suppose por contradiction that there is an algorithm $A$ that solves edge covering on $G$. As observed by Remark 2 in the proof of Lemma 4, $A$ solves edge covering on $G$ for two robots. Thus, $G$ has a cycle, by Lemma 5 . Lemma 4 implies that $A$ cannot exist.

Finally, from Lemmas 5 and 6 and Theorem 3 in "The edge-covering problem" section, we derive a full characterization for the solvability of edge covering.

Theorem 5 (Solvability of Edge Covering) For two robots, edge covering on $G$ is solvable if and only if $G$ is connected and has a simple cycle of odd length. For three or more robots, edge covering is unsolvable on any graph $G$.

\section{A topological perspective}

The topological approach to distributed computing [21] has been useful to understand the nature of fault-tolerant distributed computing, to prove impossibility results and to understand why in some case there exists a distributed algorithm to solve a problem. In this section, we briefly discuss a topological perspective of graph convergence and edge covering.

\section{The topology of graph convergence}

Our graph convergence problem is a special case of the robot convergence task defined in [21], which is specified as follows. A collection of $n$ robots are placed on the vertices of a graph $G$. The robots are asynchronous, communicate through read/write shared registers and eventually (in a wait-free manner) each one chooses a final vertex and halts. The final vertices must belong to the same edge (in the book, to the same simplex of an arbitrary complex). If they are all placed initially on the same vertex or edge, then they stay there (although they may move from one vertex to the other, even they may all move to the same vertex). If the robots are placed on vertices that do not belong to the same edge, they can move to any vertices of $G$, as long as the vertices belong to an edge.

Formally, a robot convergence task for a graph $G$ is given by a triple $(\mathcal{I}, G, \Delta)$, where $\mathcal{I}$ consists of all the subsets of $V$ of at most $n$ vertices of $G$. Such a set $\mathcal{I}$, consisting of a family of sets closed under containment is called in topology a simplicial complex. An element $\sigma$ of $\mathcal{I}$ is called a simplex, and its dimension is $|\sigma|-1$. Thus, $\sigma \subseteq V$, $|\sigma| \leq n$. For each simplex $\sigma$ in $\mathcal{I}$, representing possible simultaneously starting vertices of $G, \Delta$ encodes the convergence rules. Namely, $\Delta(\sigma)$ is a subgraph of $G$ where the robots may end up, if their initial positions are in $\sigma$. Thus, $\Delta(\sigma)=\sigma$ if $\sigma$ is either a vertex or an edge of $G$, and otherwise, $\Delta(\sigma)=G$. The following is from the book [21] (page 88) (see also [24]).

Theorem 6 (4.3.1 [21]) The graph convergence task $(\mathcal{I}, G, \Delta)$ has a wait-free n-process read/write protocol if and only if there is a continuous map $f:|\mathcal{I}| \rightarrow|G|$ carried by $\Delta$.

This theorem considers $G$ as a continuous space, denoted by $|G|$, as if $G$ was embedded in some sufficiently large Euclidean space: vertices of $G$ are points of the space, and edges are lines connecting their corresponding vertex-points. Similarly, the space $|\mathcal{I}|$ consists of the points where the vertices of $\mathcal{I}$ are placed in Euclidean space, and linear subspaces spanned by vertices belonging to the same simplex $\sigma$ of $\mathcal{I}$. The continuous map $f$ respects the input/output specification of the task, $\Delta$ (i.e., it is carried by $\Delta)$, in the sense that for each simplex $\sigma \in \mathcal{I}, f(|\sigma|)$ is in $|\Delta(\sigma)|$. In particular, $f(v)=v$, and $f(e)=e$ for any 
edge $e$. But $f$ may send a simplex $\sigma$ which is not an edge anywhere in $G$.

Theorem 6 can be used to derive simple impossibility proofs, as shown below.

Corollary 1 If $G$ is disconnected, graph convergence on $G$ is unsolvable for $n \geq 2$ robots.

Proof Let $u, v$ be vertices of different connected components, $G_{u}, G_{v}$. Consider the simplex $\sigma=\{u, v\}$ of $\mathcal{I}$. Suppose there is a solution to the task, let $f$ be its associated map by Theorem 6 . Then, $f(u)$ is in connected component $G_{u}$ and $f(v)$ is in connected component $G_{v}$. It is impossible to extend $f$ to all of $\sigma$, because $\sigma$ is connected, while $G_{u} \cup G_{v}$ is disconnected, a contradiction.

Corollary 2 If $G$ has a cycle, graph convergence on $G$ is unsolvable for $n \geq 3$ robots.

Proof Let $G$ be a graph with a cycle $C=v_{1}, v_{2}, \ldots, v_{p}, v_{1}$ (see Fig. 5 for an illustration with $p=4$ ). Then, $C$ is a cycle of $\mathcal{I}$. Once again, suppose there is a solution to the task, let $f$ be its associated map by Theorem 6 . We have that $f(C)=C$, where for each $v_{i}, f\left(v_{i}\right)=v_{i}$, and $f\left(v_{i}, v_{i+1}\right)=$ $\left(v_{i}, v_{i+1}\right)$, by the validity requirement of graph convergence. Notice that $\mathcal{I}$ is the $(n-1)$-skeleton of a simplex with vertices $V$, i.e., the set of all simplexes of dimension at most $n-1$. We can view $V$ as a complex (consisting of the set $V$ and all its faces) which represents a solid ball of dimension $|V|-1$. Hence, any cycle on $\mathcal{I}$ (its $n-1$, dimensional skeleton, with $n-1 \geq 2$ ) is contractible. Thus, $C$ is contractible in $\mathcal{I}$, but its image, $f(C)$ is not contractible, because $f(C)=C$.

The intuition for Theorem 6 becomes clear considering the effect of processes taking immediate snapshots. As shown in Fig. 1, all possible views obtained by three processes have the effect of subdividing a triangle, which in turn represent the inputs of the processes. As the immediate snapshot algorithm is repeated more and more times, finer and finer subdivisions are obtained, and hence a better approximation to a continuous map (see from [21]).

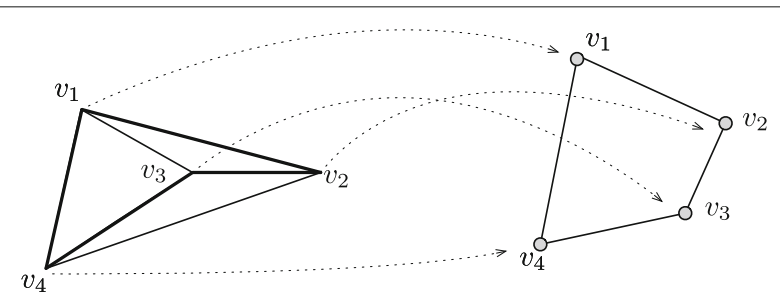

Fig. 5 Topological perspective of graph convergence. Impossibility of graph convergence on cycle $C=v_{1}, v_{2}, v_{3}, v_{4}, v_{1}$ by three processes
For more processes, subdivisions of higher dimensional simplexes are obtained.

Figure 6 illustrates how a solution is obtained for a tree with three processes, first with a continuous map, which has to then be approximated by using subdivisions (righthand side). Notice that a simplicial map from a subdivision to the output complex represents the decisions taken in each vertex.

\section{The topology of edge covering}

The underlying topological behavior of the edge covering problem is more complex, because the problem cannot be described solely by the vertices of $G$ where robots may start and may end. In addition, it is necessary to specify which robot starts or ends in which vertex. The colored version of a graph $G$ for two robots $A, B$, required to model the edge covering problem, denoted $\tilde{G}=(\tilde{V}, \tilde{E})$, consists of all pairs of vertices of the form $\langle i d, v\rangle$, where $v \in V$ and id $\in\{A, B\}$.

An edge $\left(x, x^{\prime}\right)$ belongs to $\tilde{E}$ iff $x=\langle i d, v\rangle x^{\prime}=\left\langle i d^{\prime}, v^{\prime}\right\rangle$ such that $\left(v, v^{\prime}\right) \in E\left(v \neq v^{\prime}\right)$, and $i d \neq i d^{\prime}$. A vertex $\langle i d, v\rangle$ represents the situation where robot $i d$ ends in vertex $v$.

Recall that robots have to end in adjacent vertices of $\tilde{G}$. Figure 7 illustrates that robots $A$ and $B$ have to end in vertices belonging to the same edge of $G$, but cannot both end in the same vertex.

The two-robot edge covering problem for a graph $G=$ $(V, E)$ is formally modelled as a colored task $\langle\tilde{I}, \tilde{G}, \tilde{\Delta}\rangle$, where the input graph $\tilde{I}$ is the colored version of the complete graph (including self-loops) on $|V|$ vertices. Thus, for each pair of vertices of $V,\left(v, v^{\prime}\right)$, not necessarily distinct, there is an edge $\left(x, x^{\prime}\right) \in \tilde{E}$ with $x=\langle A, v\rangle, x^{\prime}=$ $\left\langle B, v^{\prime}\right\rangle$, meaning that it is possible that $A$ starts in $v$ and $B$ starts in $v^{\prime}$. Then, the relation $\tilde{\Delta}$ is defined as follows. First, if a robot runs solo, it stays in its initial vertex, $\tilde{\Delta}(\langle i d, v\rangle)=\{\langle i d, v\rangle\}$, for every vertex $\langle i d, v\rangle$. Second, if the robots start in an edge, they stay there, i.e., $\forall\left(v, v^{\prime}\right) \in E$ :

$$
\tilde{\Delta}\left(\langle A, v\rangle,\left\langle B, v^{\prime}\right\rangle\right)=\left\{\langle A, v\rangle,\left\langle B, v^{\prime}\right\rangle,\langle B, v\rangle,\left\langle A, v^{\prime}\right\rangle\right\}
$$

Finally, if they do not start in vertices of the same edge, they can decide on any vertices belonging to the same edge, i.e., $\forall\left(v, v^{\prime}\right) \notin E, \tilde{\Delta}\left(\langle A, v\rangle,\left\langle B, v^{\prime}\right\rangle\right)=\tilde{G}$.

The wait-free solvability theorem [25] implies that the edge covering problem for two robots has a solution if and only if there is a subdivision $X$ of $\tilde{I}$ and a simplicial map $\delta$ from $X$ to $\tilde{G}$, such that $\delta$ preserves ids and edge adjacencies, and $\delta$ respects $\tilde{\Delta}$. It is called a decision map because it represents the outputs of the distributed algorithm that the robots execute. Namely, when the robots start in an edge $\left(x, x^{\prime}\right) \in \tilde{I}, x=\langle A, v\rangle$ and $x^{\prime}=\left\langle B, v^{\prime}\right\rangle$, it is known that the distributed algorithm induces a subdivision of $\left(x, x^{\prime}\right)$, essentially creating a path, where each edge 


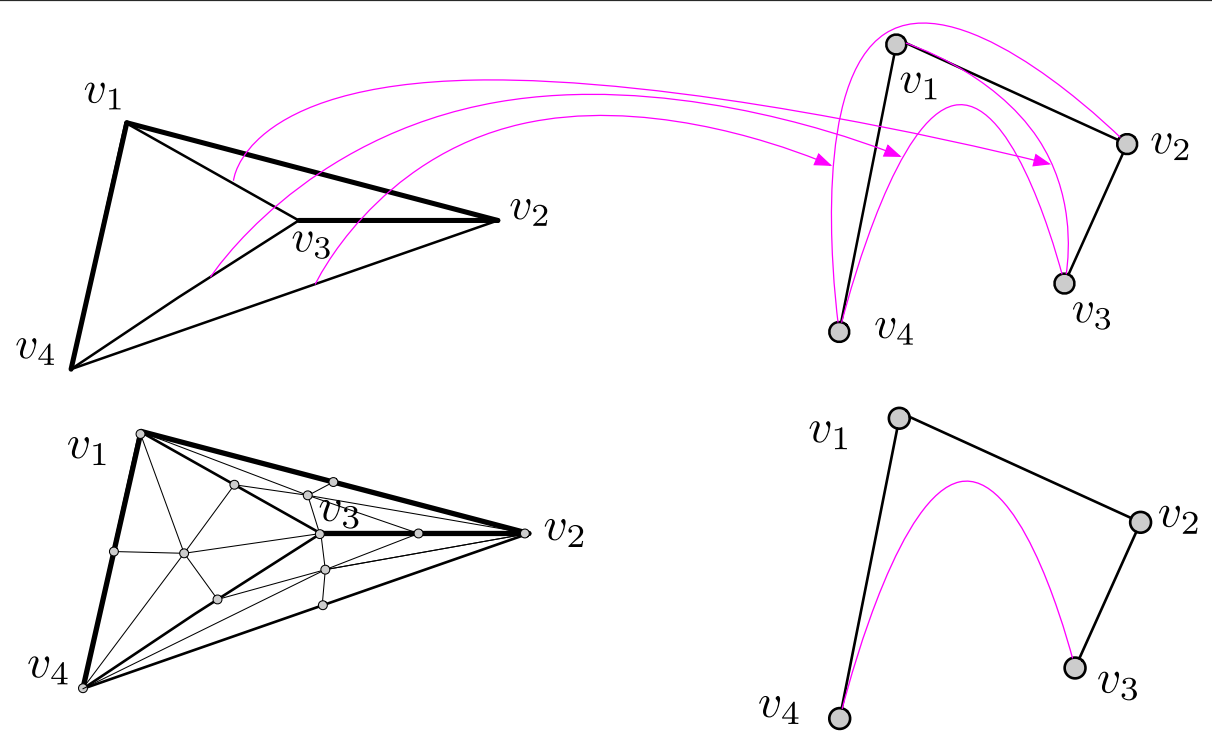

Fig. 6 Topological perspective of graph convergence. Top Solving a tree by three processes. Bottom Trying to solve a tree in one round

of the path represents the final states of the robots in one of the possible executions starting in $\left(x, x^{\prime}\right)$.

Theorem 7 (3.1 [25]) A task $(\mathcal{I}, \mathcal{O}, \Delta)$ has a wait-free read/write protocol iff there is a chromatic subdivision $X$ of $\mathcal{I}$ and a color-preserving simplicial map $\delta: X(\mathcal{I}) \rightarrow \mathcal{O}$ s.t. for each $\sigma \in X(\sigma), \delta(\sigma)$ is carried by $\Delta(\sigma)$ (see Fig. 8).

A consequence is that the task has a solution for $A, B$ iff for any two vertices of $G$ there is an odd length path, corresponding to a path in $\tilde{G}$ alternating vertices with id $A$ and $B$.

Notice that the formal specification of the edge covering problem as a triple $\langle\tilde{I}, \tilde{G}, \tilde{\Delta}\rangle$, depends on the number of robots, and indeed we defined it above for $A, B$. To go beyond two robots, to three robots, it is necessary to add to triangles to the graphs, representing positions of three robots $A, B, C$, and more generally, simplices of $n$ vertices, labeled with distinct robot ids. Then, the wait-free solvability theorem [25] is about general dimension combinatorial topology simplicial complexes. Roughly speaking, edge covering (and in general non-colorless tasks) is more difficult that graph convergence, because of a seemingly innocuous, but surprisingly "difficult" requirement: the simplicial decision map $\delta$ is color-preserving. Namely, while in a colorless task, robots can always adopt each other outputs ( $\delta$ can send simplexes to lower dimensional output simplexes), this is not possible in general tasks $(\delta$ sends a final state's algorithm simplex to an output simplex of the same dimension).

\section{Round-complexity optimality}

Once the topology of the graph convergence and edge covering is understood, it is easy to argue that our algorithms in previous sections are asymptotically round-complexity optimal.

For graph convergence, consider the simple case in which two robots, say $p_{u}$ and $p_{v}$, start on vertices $u$ and $v$ at distance $\operatorname{diam}(G)$. As explained above, this initial configuration is represented with an edge, $S^{0}$, with its vertices representing the processes initially standing on those vertices. After a first round, this line is subdivided into three edges, $S^{1}$, each of them modeling the three possible immediate snapshots: $p_{u}$ goes first or viceversa, or $p_{u}$ and $p_{v}$ go together. For example, the bottom edges in the subdivision in Fig. 1 correspond to the immediate snapshot for robots 2 and 3 . In a second round, each of these three edges is subdivided again into three edges to obtain a subdivision, $S^{2}$, with nine edges, and so on. In general, the subdivision $S^{r}$ of the edge $S^{0}$ obtained after $r$ rounds has

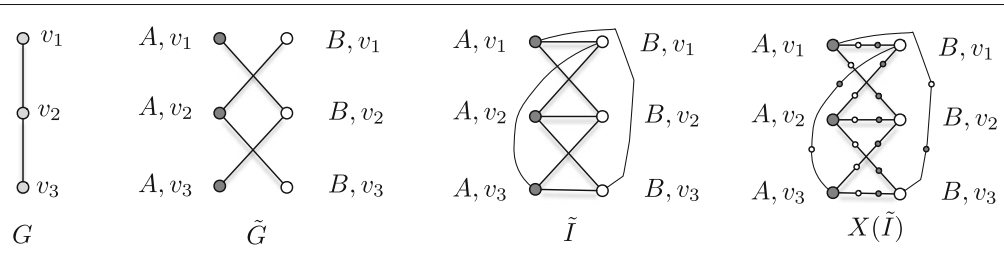

Fig. 7 Topological perspective of edge-covering. $G$ and colored graph $\tilde{G}$, with input $\tilde{I}$ and views after one round $X(\tilde{I})$, for robots $A, B$ 

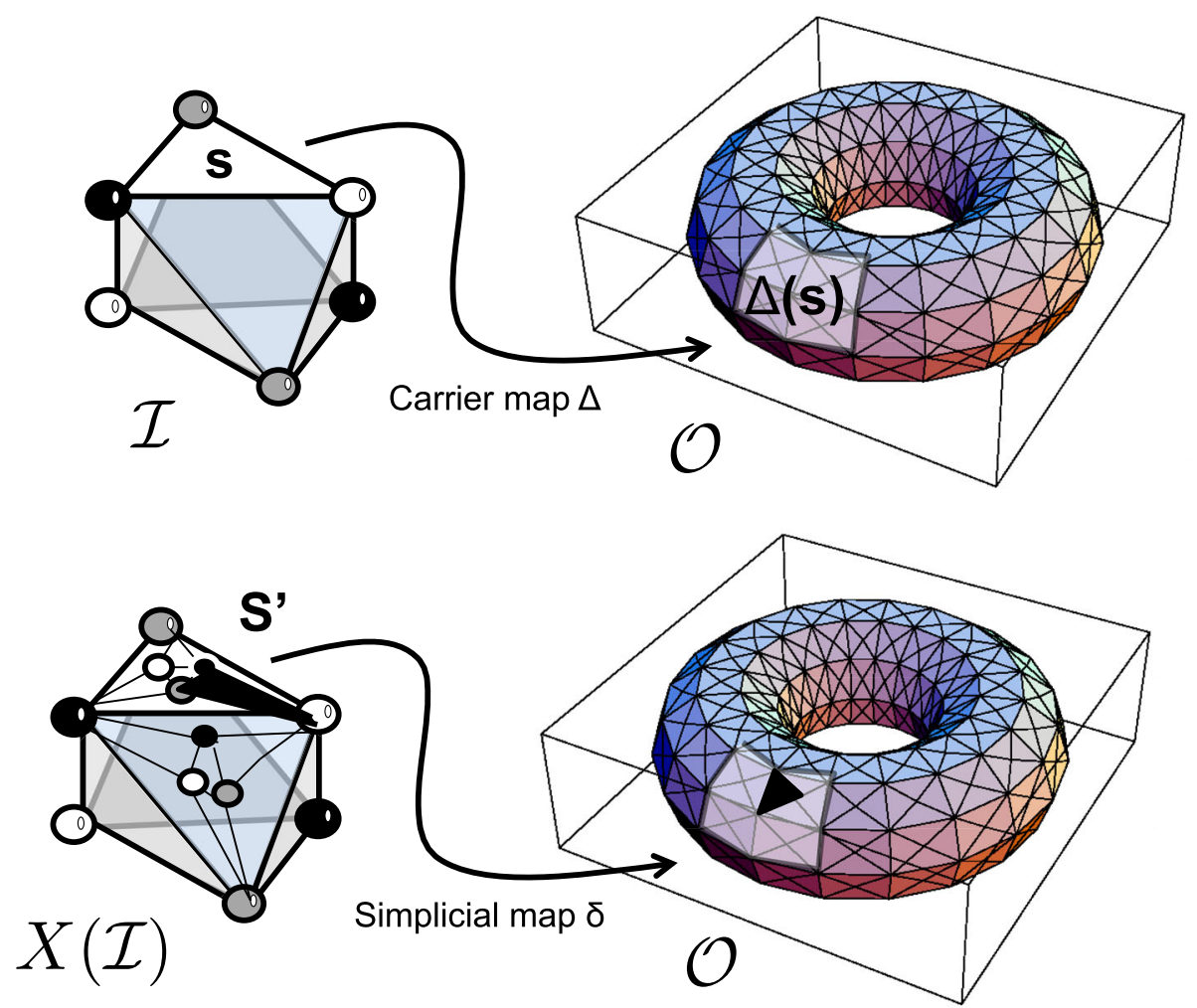

Fig. 8 The wait-free computability theorem. A graphical description of the wait-free computability theorem

$3^{r}$ edges. Thus, the length of the subdivision exponentially grows on the number of rounds, which implies that after $O(\log \operatorname{diam}(G))$ rounds the length of $S^{O(\log \operatorname{diam}(G))}$ is $\Omega(\operatorname{diam}(G))$.

Now, due to the agreement property of graph convergence, any algorithm that solves this problem must map the vertices in $S^{O(\log \operatorname{diam}(G))}$ to vertices of $G$ satisfying that the vertices of any edge are mapped to vertices of $G$ at distance at most 1 . Moreover, the validity property implies that the extreme vertices of the subivision must be mapped to $u$ and $v$ : these vertices in $S^{O(\log \operatorname{diam}(G))}$ represent the solo executions of the processes, one of $p_{u}$ and one of $p_{v}$, and the validity property states that in solo executions a process must decides its initial vertex. Thus, the extreme vertices of $S^{O(\log \operatorname{diam}(G))}$ are mapped to $u$ and $v$ and all other vertices are mapped to vertices of $G$, satisfying the agreement property. In other words, $S^{O(\log \operatorname{diam}(G))}$ is mapped to a path of $G$ from $u$ to $v$ where each edge of the subdivision is either mapped to an edge of the path or "collapsed" to a vertex of the path. This mapping is possible because the length of $S^{O(\log \operatorname{diam}(G))}$ is $\Omega(\operatorname{diam}(G))$, as explained above.

It is now easy to see that if robots perform only $o(\log \operatorname{diam}(G))$ rounds, they cannot solve graphconvergence: in such a case the diameter of $S^{o(\log \operatorname{diam}(G))}$ is $o(\operatorname{diam}(G))$, and hence it is impossible to map
$S^{o(\log \operatorname{diam}(G))}$ to a path of $G$ from $u$ to $v$ as described above. Hence, the round-complexity optimality of our graph-convergence algorithms.

The analysis for edge covering is essentially the same, the only difference is that, due to the agreement property of the problem, each edge of the subdivision must be mapped to an edge of $G$. From this perspective, we can say more. It is needed the existence of an odd-length path in $G$ from $u$ to $v$ : $S^{O(\log \operatorname{diam}(G))}$ is mapped to a path in $G$ from $u$ to $v$, and each edge of the subdivision is mapped to an edge of the path; however, as explained above, the length of $S^{O(\log \operatorname{diam}(G))}$ is odd (more precisely, $3^{O(\log \operatorname{diam}(G))}$ ), from which follows that the length of the path $S^{O(\log \operatorname{diam}(G))}$ is mapped to must be odd.

\section{Conclusion}

In this paper, we study two robot convergence problems in an asynchronous read/write shared memory crash-prone system, where the base space is a finite graph. The problems are the graph convergence (robots decide vertices that belong to the same edge) and edge covering (robots decide vertices that cover an edge). For both tasks we show possibility and impossibility results that fully characterize the graphs on which these problems can be solved. Additionally, we give a topological perspective of the solvability of both problems. 
The study of the two robot convergence problems presented in this paper is of a theoretical nature. The purpose is to understand which problems are in principle solvable, and show inherent limitations of what is possible. We show that the two robot convergence problems are unsolvable if $G$ has a cycle, when the number of robots is at least 3 (Lemma 4). This implies an inherent limitation about robot coordination, even if the robots know $G$ and can communicate with each other reliably. The limitation comes from the fact that the robots are asynchronous and they may crash. This means that if one abstracts away a two-dimensional space that has a hole that must be avoided by the robots by a graph, the tasks are unsolvable when $n \geq 3$. Another contribution, is to expose the inherent difficulty of requiring the robots to coordinate to cover an edge: edge covering is unsolvable when the number of robots is at least 3 (Lemma 6). Indeed, edge covering is a more subtle problem. We have shown that for two robots, solving it requires the existence of an odd length cycle (Lemma 5).

Although the study presented is theoretical, we hope it motivates to solve solutions to robot convergence problems in realistic settings. For the cases we provide impossibility results, it would be interesting to search for probabilistic solutions. A limitation of our model is that robots communicate reliably using a shared memory, see the exact positions of other robots, and may jump directly to any vertex. In reality robots may have limited visibility of other robots and see only their approximate positions. We investigate a more detailed model where robots can move only to adjacent vertices in $G$, in [2]. Extending our algorithms to such situations requires interesting future research. Also, we consider only crash failures. Interesting future work may consider Byzantine failure techniques such as [28]. In contrast, we expect that the assumption that robots have identifiers is common in practice. In any case, our algorithms do not make an essential use of identifiers.

We assume that robots are located on a space that can be modelled as a graph. Some spaces encountered in applications can be naturally modeled as graph, or a graph can be extracted, see [29] for a survey of techniques for extracting graph representations of the environment. Further research is needed to extend our results to two or even three dimensional spaces. We expect that more sophisticated topological techniques may be needed.

Our two-robot convergence tasks are abstractions that capture the fundamental difficulty of coordination. Understanding the inherent difficulty in coordination and providing sound and proven solutions is a prerequisite for designing algorithms for real applications; many reallife situations in distributed robotics, such as cooperative vehicular communication, or rescue missions, require that robots coordinate to get to positions close to each other.
In practice, robots do not have a physical shared memory to communicate with each other; typically, they communicate through a weaker communication medium, sending messages to each other, observing each other, etc. The model we consider can be simulated on top of a system where processes communicate by sending messages to each other, where a majority of them do not crash [3]. Thus, all our impossibility results hold also in that setting and our algorithms can be translated to such network system using the simulation in [3].

\section{Funding}

The first author is supported by UNAM-PAPIIT IA102417. The second author is supported by UNAM-PAPIIT IN109917 and also received support from ECOS-CONACYT and LAISLA. The third author is supported by CPSLab project H2020-ICT-644400. The first and second authors want to thank INRIA for its support in the context of the INRIA-UNAM "Équipe Associée" LiDiCo (At the Limits of Distributed Computing).

\section{Availability of data and materials}

Not applicable.

Authors' contributions

All authors read and approved the final manuscript.

Ethics approval and consent to participate

Not applicable.

Consent for publication

Not applicable.

Competing interests

The authors declare that they have no competing interests.

\section{Publisher's Note}

Springer Nature remains neutral with regard to jurisdictional claims in published maps and institutional affiliations.

\section{Author details}

${ }^{1}$ Instituto de Matemáticas, UNAM, Ciudad Universitaria, 04510 Mexico city, Mexico. ${ }^{2}$ LAAS-CNRS, 7, av du Colonel Roche, 31077 Toulouse, France.

Received: 19 May 2017 Accepted: 6 December 2017

Published online: 08 January 2018

\section{References}

1. Agmon N, Peleg D (2006) Fault-tolerant gathering algorithms for autonomous mobile robots. SIAM J Comput 36(1):56-82

2. Alcántara M, Castañeda A, Peñaloza DF, Rajsbaum S (2017) Fault-tolerant robot gathering problems on graphs with arbitrary appearing times. In: 2017 IEEE International Parallel and Distributed Processing Symposium (IPDPS). IEEE. pp 493-502

3. Attiya H, Bar-Noy A, Dolev D (1995) Sharing memory robustly in message-passing systems. J ACM 42(1):124-142

4. Attiya H, Bar-Noy A, Dolev D, Peleg D, Reischuk R (1990) Renaming in an asynchronous environment. J ACM 37(3):524-548

5. Attiya H, Welch J (2004) Distributed computing: fundamentals, simulations, and advanced topics. Wiley

6. Bhattacharya S, Lipsky D, Ghrist R, Kumar V (2013) Invariants for homology classes with application to optimal search and planning problem in robotics. Ann Math Artif Intell 67(3):251-281

7. Biran O, Moran S, Zaks S (1990) A combinatorial characterization of the distributed 1-solvable tasks. J Algoritm 11(3):420-440

8. Borowsky E, Gafni E, Lynch N, Rajsbaum S (2001) The BG distributed simulation algorithm. Distrib Comput 14(3):127-146

9. Borowsky E, Gafni E (1993) Generalized FLP impossibility result for t-resilient asynchronous computations. In: Proceedings of the 
Twenty-Fifth Annual ACM Symposium on Theory of Computing, May 16-18, 1993, San Diego, CA, USA. pp 91-100

10. Bouzid Z, Das S, Tixeuil S (2013) Gathering of mobile robots tolerating multiple crash faults. In: Proceedings of the 2013 IEEE 33rd International Conference on Distributed Computing Systems, ICDCS '13. IEEE Computer Society, Washington. pp 337-346

11. Castañeda A, Rajsbaum S (2012) New combinatorial topology bounds for renaming: the upper bound. J ACM 59(1):3:1-3:49

12. Castañeda A, Rajsbaum S, Raynal M (2011) The renaming problem in shared memory systems: an introduction. Comput Sci Rev 5(3):229-251

13. Castañeda A, Imbs D, Rajsbaum S, Raynal M (2016) Generalized symmetry breaking tasks and nondeterminism in concurrent objects. SIAM J Comput 45(2):379-414

14. Chaudhuri S (1993) More choices allow more faults: set consensus problems in totally asynchronous systems. Inf Comput 105(1):132-158

15. Das S, Flocchini P, Prencipe G, Santoro N, Yamashita M (2016) Autonomous mobile robots with lights. Theor Comput Sci 609(P1):171-184

16. Dolev D, Lynch NA, Pinter SS, Stark EW, Weihl WE (1986) Reaching approximate agreement in the presence of faults. J ACM 33(3):499-516

17. Fischer MJ, Lynch NA, Paterson M (1985) Impossibility of distributed consensus with one faulty process. J ACM 32(2):374-382

18. Gafni E, Rajsbaum S (2010) Recursion in distributed computing In: Proceedings of the 12th International Conference on Stabilization, Safety, and Security of Distributed Systems, SSS'10. Springer-Verlag, Heidelberg. pp 362-376

19. Gafni E, Rajsbaum S, Herlihy M (2006) Subconsensus tasks: renaming is weaker than set agreement. In: Proceedings of the 20th International Conference on Distributed Computing, DISC'06. Springer-Verlag, Heidelberg. pp 329-338

20. Herlihy M (1991) Wait-free synchronization. ACM Trans Program Lang Syst 13(1):124-149

21. Herlihy M, Kozlov D, Rajsbaum S (2013) Distributed computing through combinatorial topology, 1st edition. Morgan Kaufmann Publishers Inc., San Francisco

22. Herlihy M, Rajsbaum S (1997) The decidability of distributed decision tasks (extended abstract). In: Proceedings of the Twenty-ninth Annual ACM Symposium on Theory of Computing, STOC '97. ACM, New York. pp 589-598

23. Herlihy M, Rajsbaum S (2003) A classification of wait-free loop agreement tasks. Theor Comput Sci 291(1):55-77

24. Herlihy M, Rajsbaum S, Raynal M, Stainer J (2014) LATIN 2014: Theoretical Informatics: 11th Latin American Symposium, Montevideo, Uruguay, March 31-April 4, 2014. In: Proceedings, chapter Computing in the Presence of Concurrent Solo Executions. Springer Berlin Heidelberg, Heidelberg. pp 214-225

25. Herlihy M, Shavit N (1999) The topological structure of asynchronous computability. J ACM 46(6):858-923

26. Hoest G, Shavit N (2006) Toward a topological characterization of asynchronous complexity. SIAM J Comput 36(2):457-497

27. Liu X, Xu Z, Pan J (2009) Classifying rendezvous tasks of arbitrary dimension. Theor Comput Sci 410(21-23):2162-2173

28. Mendes H, Herlihy M, Vaidya N, Garg VK (2015) Multidimensional agreement in byzantine systems. Distrib Comput 28(6):423-441

29. Portugal D, Rocha RP (2013) Retrieving topological information for mobile robots provided with grid maps, Number 358 in Communications in Computer and Information Science. Springer Berlin Heidelberg, Heidelberg

30. Saraph V, Herlihy M The relative power of composite loop agreement tasks. In: Proceedings of the International Conference on Principles of Distributed systems (OPODIS), LIPIcs: Leibniz Int. Proc. Informatics, Germany, 2015. Dagstuhl

\section{Submit your manuscript to a SpringerOpen ${ }^{\circ}$ journal and benefit from:}

- Convenient online submission

- Rigorous peer review

- Open access: articles freely available online

- High visibility within the field

- Retaining the copyright to your article

Submit your next manuscript at $\gg$ springeropen.com 